'Instituto de Actividad Física y Salud, Universidad Autónoma de Chile, Chile.

2Escuela de Ciencias de la Salud, Universidad Católica de Temuco, Temuco, Chile. ${ }^{3}$ Facultad de Ciencias del Deporte, Universidad de Extremadura, España. ${ }^{a}$ MSc Motricidad Humana. ${ }^{\mathrm{b}} \mathrm{MSc}$ Salud Publica. CPhD Ciencias de la Actividad Física y el Deporte. dMSc Investigación en Ciencias de la Actividad Física y el Deporte.

Recibido el 29 de abril de 2015, aceptado el 5 de agosto de 2015 .

Correspondencia a: Pedro R. Olivares Facultad de Educación. Universidad Autónoma de Chile. 5 Poniente 1670, Talca, Chile. +(56) (71) 2735705 polivaress@uautonoma.cl

\section{Traducción y adaptación cultural del cuestionario de independencia física Composite Physical Function para su uso en Chile}

\author{
EUGENIO MERELLANO-NAVARRO ${ }^{1, \mathrm{a}}$, MICHELLE LAPIERRE ${ }^{2, \mathrm{~b}}$, \\ JAVIER GARCÍA-RUBIO ${ }^{1, c}$, NARCÍS GUSI ${ }^{3, c}$, \\ DANIEL COLLADO-MATEO ${ }^{3, \mathrm{~d}}$, PEDRO R. OLIVARES ${ }^{1, \mathrm{c}}$
}

\section{Translation and cultural adaptation of the Composite Physical Function for its use in Chile}

Aging is directly related with loss of physical independency. Composite Physical Function questionnaire (CPF) assess, throw 12 items, a range of daily life activities in order to determine dependency levels in elderly. However, there is not a Spanish version of this instrument. Aim: To translate and culturally adapt the CPF to Spanish for its use in Chilean elderly. Method: Standardized international methodology was used in this study, which consisted in double direct translation to Spanish, harmonization of versions and back-translation to English. Acceptability and familiarity of the obtained version was analyzed using probing and paraphrasing methods using a sample of 20 older adults aged from 65 to 80 years old. Results: All items were clear and understandable, although minor adaptations needed to be done in order to improve the understandability of two items. These adaptations consisted in adding information in brackets at the end of the sentence. Conclusion: Spanish version of the CPF questionnaire was obtained to its use in Chile. This questionnaire has been proved to be understandable and adapted to its use in Chilean older adults. Its ease of use makes this questionnaire potentially useful in future researches and surveys.

(Rev Med Chile 2015; 143: 1314-1319)

Key words: Elderly; Questionnaires; Transcultural study.

\footnotetext{
$\mathrm{E}$
} 1 proceso de envejecimiento conlleva la pérdida de las capacidades funcionales de forma progresiva acorde a la edad, acelerando de forma considerable una vez que se sobrepasa el umbral de los 80 años $^{1}$. La calidad de vida está directamente relacionada con la preservación de la movilidad y la independencia física funcional en edad adulta ${ }^{2}$. Evaluar los niveles de independencia en las actividades de la vida diaria se hace necesario a la hora de diseñar programas de trabajo y estrategias dirigidas al adulto mayor para mejorar su salud y calidad de vida. Existe un número considerable de instrumentos que se utilizan para medir el nivel de independencia funcional en adultos mayores, los cuales suelen requerir mucho tiempo durante su evaluación, e incluso muchos de ellos la presencia de personal cualificado. El cuestionario Composite Physical Function (CPF), desarrollado por Rikli y Jones ${ }^{3,4}$, se puede utilizar como auto-reporte y consta de 12 ítems que miden una amplia gama de actividades de la vida diaria, catalogadas como actividades básicas, intermedias y avanzadas. Su sencillez de uso y aplicabilidad hacen de él un instrumento 
útil para la evaluación de la independencia en la práctica clínica como herramienta de detección o screening y para estudios con metodologías epidemiológicas tales como encuestas de salud para organismos públicos.

Sin embargo, actualmente tan sólo existe en inglés (versión original) ${ }^{3,4}$ y portugués 5 . Para su uso en población de habla hispana se hace necesario su traducción y adaptación cultural, siguiendo la metodología estandarizada para tal fin.

El objetivo de este trabajo fue realizar la traducción y adaptación al español del cuestionario CPF para su uso con adultos mayores chilenos.

\section{Material y Método}

Para la traducción y adaptación cultural del CPF se siguió la metodología estandarizada a nivel internacional ${ }^{6,7}$, consistente en traducción por más de un traductor, armonización, retro-traducción y entrevistas cognitivas (Figura 1).

\section{Proceso de traducción y armonización}

En esta primera fase, dos investigadores con el español como lengua nativa, experiencia en cuestionarios de adultos mayores y dominio del idioma original del instrumento (inglés), realizaron, de forma simultánea e independiente, una primera propuesta de adaptación de la versión internacional del cuestionario CPF. Los dos traductores, junto con otro miembro del grupo de investigación, contrastaron ambas traducciones con el fin de crear una versión conciliada en español, que conservara una óptima equivalencia semántica y conceptual de la versión internacional de este cuestionario.

Posteriormente, la versión conciliada en español fue sometida a un proceso de retro traducción al inglés por parte de un nativo del idioma inglés y con dominio del español. Un investigador externo con experiencia en procesos de traducción realizó una comparación de la versión original del CPF con la versión resultante de la retro traducción, con el objetivo de identificar discrepancias entre las versiones. De esta forma, se obtuvo la segunda versión consensuada.

\section{Entrevistas cognitivas}

La versión en español desarrollada fue evaluada en los ámbitos de comprensión, claridad

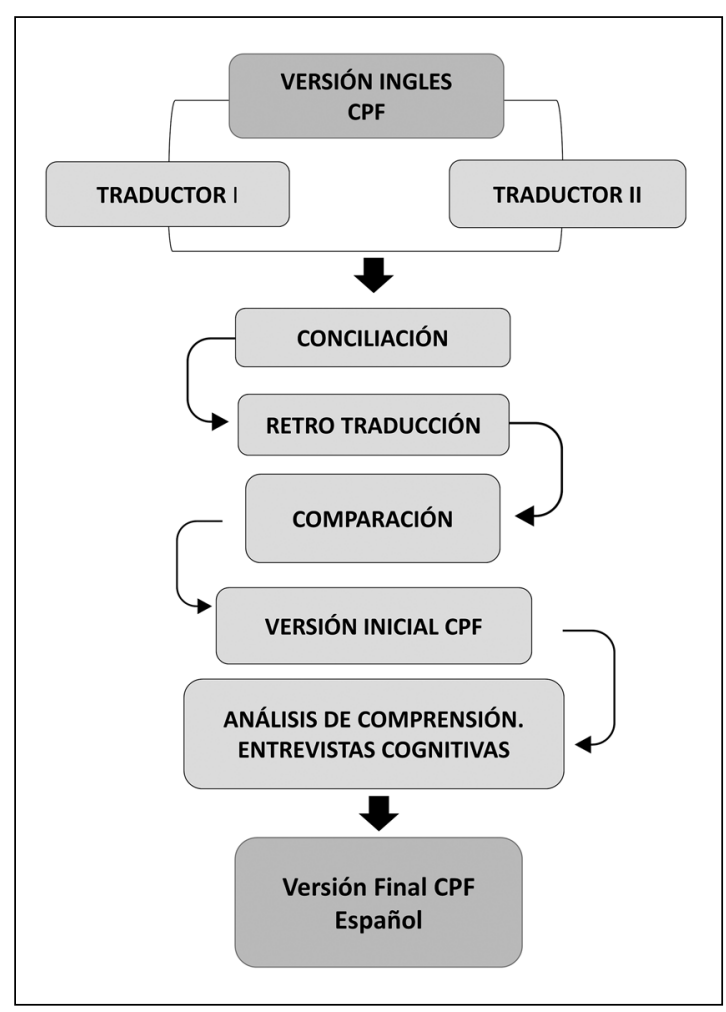

Figura 1. Proceso de traducción y adaptación del instrumento.

y familiaridad de los conceptos por medio de entrevistas cognitivas con los métodos de indagación y parafraseo. Estas entrevistas consistieron en evaluación de la comprensión en escala ordinal: los entrevistados debían evaluar cada ítem mediante una escala de 1 a 7 , siendo 7 la máxima comprensión y 1 nula comprensión. Para evaluar la indagación y parafraseo se empleó la estrategia de pedirles a los entrevistados que expresaran con sus propias palabras el significado percibido y la redacción más lógica para ellos de cada ítem. Todas las entrevistas se revisaron de forma individual y oral. Las entrevistas cognitivas se realizaron con una muestra de conveniencia formada por un total de 20 adultos mayores de las ciudades de Talca y Santiago (Chile), con una edad comprendida entre 65 y 83 años. Se establecieron los siguientes criterios de inclusión: adulto mayor de 65 años, sin diagnóstico de demencia, que viva en la comunidad y con participación activa en agrupaciones comunitarias de adulto mayor. El reclutamiento de la muestra se realizó mediante 
el contacto con una agrupación de adulto mayor de la ciudad de Talca y otra de la ciudad de Santiago. El estudio cumple con los principios de la Declaración de Helsinki y sus revisiones posteriores para estudios en humanos. Todos los participantes fueron informados de los objetivos del estudio previamente a su inclusión en él y aceptaron los términos del mismo firmando un documento de consentimiento informado.

\section{Resultados}

Todos los participantes evaluaron el instrumento como claro y comprensible, puntuando con 7 sobre 7 en comprensión, en 10 de los 12 ítems. Dos ítems (uno relacionado con la práctica de senderismo y otro referido a caminar un número determinado de cuadras) se calificaron con un promedio de 5. Los participantes de mayor edad fueron los que indicaron una menor comprensión, aunque siempre con valores de 5 sobre 7 o más. Con las técnicas de indagación y parafraseo se comprobó que todos los entrevistados lograban comprender correctamente los 12 ítems del cuestionario, aunque en los dos anteriormente indicados hubo que intervenir para lograr la comprensión correctamente.

\section{Discusión}

En este estudio se ha aportado la traducción y adaptación para su uso en Chile del cuestionario de independencia física $\mathrm{CPF}$, el cual permitirá una rápida detección de personas con problemas de dependencia física durante la práctica clínica. Además, debido a su sencillez y rápida aplicación podrá ser utilizado en futuros estudios que necesiten evaluar la dependencia en amplias poblaciones, e incluso en encuestas poblacionales.

La metodología utilizada en la traducción al español del CPF para su uso en adultos mayores chilenos asegura la equivalencia semántica de estos instrumentos en distintos idiomas y culturas, permitiendo estudios transculturales y de carácter internacional ${ }^{8,9}$.

Las entrevistas cognitivas indicaron poca claridad en los ítems de esfuerzo extenuante, específicamente desconocimiento de 12 de los 20 participantes del concepto "senderismo". Se determinó, por medio de la técnica de parafraseo e indagación, que son pocos los sujetos que la asocian a caminata en áreas naturales. Por tanto, se decidió añadir al final de este ítem, y entre paréntesis, caminata en áreas naturales, para facilitar su comprensión. En los ítems referidos a distancias, producto de la traducción, se creó una confusión al buscar la equivalencia de las distancias en millas y el concepto de bloques (versión en inglés) a metros y cuadras (versión español contextualizado a Chile). Se decidió aproximar las distancias a valores en metros y señalar su aproximación en cuadras (calculado 1 cuadra $=100$ metros).

Al comparar el CPF con otros instrumentos de medición de la independencia en actividades de la vida diaria (AVD), asociada a las características físicas de las cuales éstas dependan, se pueden encontrar diversos aspectos de interés. En primer lugar es necesario mencionar que el CPF busca medir el nivel de desempeño o funcionamiento (alto, moderado, bajo), entregando una visión general del resultado final del adulto mayor. Otros instrumentos que evalúan desempeño general en AVD son el Índice de Barthel ${ }^{10}$ y el Índice de Katz ${ }^{11}$, los cuales entregan también resultados de un desempeño general, evaluando el nivel de dependencia desde independiente hasta dependiente total. Sin embargo, ambos instrumentos evalúan solamente AVD básicas, a diferencia del CPF que integra, además, AVD instrumentales, ampliando el rango de evaluación y las posibilidades de ser más efectivo en un adulto mayor viviendo en comunidad. Por tanto, este instrumento puede ser más útil en adultos mayores con riesgo de perder autovalencia o con enfermedades crónicas que no son altamente discapacitantes, generando una posibilidad de evaluación de prevención primaria y secundaria, a diferencia de los otros dos instrumentos mencionados, que se enfocan en usuarios de mayor nivel de complejidad ${ }^{12}$.

En relación al tipo de AVD evaluadas, la mayoría de los instrumentos validados para su uso en Chile evalúan actividades básicas o instrumentales en forma diferenciada, principalmente porque los instrumentos se han utilizado mucho en contextos residenciales, con pacientes institucionalizados o adultos mayores semipostrados o postrados que viven en sus hogares. En este aspecto, la mayoría de los instrumentos que 
evalúan AVD instrumentales, dentro de la gama de actividades consideradas, tal como el CPF, enfatizan en la función cognitiva asociada a tales actividades y no en las motoras, a diferencia del CPF. En este sentido, el test de Pfeffer ${ }^{13}$, el cual mide AVD instrumentales más relacionadas con la cognición, aunque tiene objetivos similares, es reportado por un informante cuidador y no como auto-reporte, con las debilidades que esto puede tener, considerando el fenómeno de la sobreprotección, la cual es muy común en la cultura latinoamericana por parte de familiares e instituciones, así como la victimización por parte del usuario, como puede verse en otros casos $^{14,15}$. En este sentido, el CPF resulta un aporte tanto para facilitar la medición de ambas áreas, como para diversificar la población sujeto de evaluación, cada vez más participante de los procesos comunitarios y ciudadanos. Existen instrumentos que evalúan una gran gama de AVD tanto básicas como instrumentales, tales como el Functional Independence Measurement (FIM) ${ }^{16}$, sin embargo, su aplicación no es comparable, dado que no es un instrumento auto-administrado y requiere una capacitación y certificación especializada por parte del personal de salud ${ }^{16}$, lo cual aventaja al $\mathrm{CPF}$ en relación a la simplicidad de gestión para la aplicación.

En un tercer aspecto de análisis, el CPF es un instrumento que demostró ser de fácil comprensión y adaptación al idioma y la cultura, como se ha mencionado anteriormente. Sin embargo, otros instrumentos ampliamente utilizados como el Índice de Barthe ${ }^{10}$ y el Índice de Katz ${ }^{11}$ presentan mayores detalles para poder explicar las diversidad de opciones que pueden haber asociado a las actividades en particular y las diferencias de desempeño, lo cual resulta una fortaleza al comparar con la sobriedad y concisión del CPF para realizar el planteamiento de cada punto ${ }^{17}$. El instrumento FIM se realiza mediante entrevista para indagar opciones personales que dan relevancia a variaciones culturales, geográficas y de contexto socioeconómico que sean de consideración para el análisis ${ }^{18}$. Sin embargo, todas estas opciones dificultan el rápido análisis y posibilidad de tamizaje que sí tiene el CPF.

Finalmente, el CPF enfatiza en su capacidad de ajustar sus resultados por rangos etarios, lo cual resulta no ser un elemento común en instrumentos que miden capacidad física para independen- cia, pues culturalmente se define y comprende la independencia asociada a la funcionalidad física de un modo bastante universal, centrado en la capacidad de ejecución sin asistencia y que se asume en declive con el aumento de edad ${ }^{19}$. Esto puede ser un aspecto positivo si es posible comparar entre otros adultos mayores de la misma edad. Sin embargo, no resulta de gran utilidad al comparar en general con otros adultos mayores de diversas edades, ni cuando se utiliza como elemento para definir la necesidad de apoyo a la dependencia o si se califica a un adulto mayor para ingreso a una institución, recibir tratamiento o beneficios estatales.

Es posible concluir que, al comparar el CPF con otros instrumentos, entrega importantes fortalezas relacionadas con la capacidad de auto-administración, medición tanto de AVD básicas como instrumentales, capacidad comparativa con pares adultos mayores y como instrumento de tamizaje y con rápido procesamiento de datos. En relación a las debilidades comparativas, se sugiere que se integren más posibilidades de variar actividades, dar mayor relevancia al contexto y las diferencias culturales que pueden interferir con los resultados $\mathrm{y}$, por último, que se pueda ampliar la capacidad de interpretación de resultados en sus categorías finales.

\section{Limitaciones}

El estudio se realizó con adultos mayores pertenecientes a zonas urbanas (Santiago y Talca) y no se consideró el sector rural. El alto índice de comprensión que obtuvieron los cuestionarios se puede deber al nivel de comprensión lectora que poseen, producto de su nivel de escolaridad. Los resultados de la evaluación de comprensión podrían ser diferentes si se hubiera considerado el ámbito rural. Sin embargo, la sencillez de los ítems que lo componen y las adaptaciones realizadas al contexto cultural chileno hacen pensar una alta comprensión también en zonas rurales.

Se espera que este instrumento sea útil para la valoración de la independencia física en población adulta chilena, así como su posible inclusión en futuros estudios de investigación e incluso en encuestas poblacionales como la encuesta nacional de calidad de vida en la vejez. No obstante, aunque este instrumento ya ha sido publicado en otras poblaciones, aún debe someterse a la comprobación de su validez y fiabilidad en población chilena. 
En conclusión, se ha obtenido la versión en español del cuestionario de independencia física CPF adaptado a Chile, el cual ha mostrado ser claro y comprensible por parte de los adultos mayores chilenos.
Agradecimientos: Los autores agradecen la colaboración de los participantes en el proceso de entrevistas cognitivas. El autor DCM agradece a la fundación Tatiana Pérez de Guzmán el Bueno por la financiación recibida en forma de contrato pre-doctoral.

\section{Composite Physical Function}

Nombre

Por favor, indique su capacidad para realizar cada una de las siguientes tareas. (Marque la respuesta adecuada)

\begin{tabular}{|c|c|c|c|}
\hline & $\begin{array}{l}\text { Puedo hacerlo } \\
\text { sin ayuda }\end{array}$ & $\begin{array}{c}\text { Puedo hacerlo } \\
\text { con ayuda }\end{array}$ & $\begin{array}{c}\text { No puedo } \\
\text { hacerlo }\end{array}$ \\
\hline A. Realizar actividades de cuidado personal básicas (como vestirse) & 2 & 1 & 0 \\
\hline B. Bañarse (usando tina o ducha) & 2 & 1 & 0 \\
\hline C. Subir y bajar escaleras (Como a un segundo piso de una casa) & 2 & 1 & 0 \\
\hline D. Caminar una o dos cuadras & 2 & 1 & 0 \\
\hline $\begin{array}{l}\text { E. Hacer tareas domésticas ligeras (tales como cocinar, sacudir, lavar } \\
\text { loza, barrer) }\end{array}$ & 2 & 1 & 0 \\
\hline F. Hacer mis compras de supermercado o ropa & 2 & 1 & 0 \\
\hline G. Caminar 750 metros (aprox. 6 a 7 cuadras) & 2 & 1 & 0 \\
\hline H. Caminar 1 1 $1 / 2$ kilometro (aprox. 15 cuadras) & 2 & 1 & 0 \\
\hline I. Levantar y transportar 5 kilos (bolsa llena del supermercado) & 2 & 1 & 0 \\
\hline J. Levantar y transportar 12 kilos (Maleta mediana o grande) & 2 & 1 & 0 \\
\hline $\begin{array}{l}\text { K. Hacer las tareas domésticas más pesadas (como trapear, pasar la } \\
\text { aspiradora, barrer las hojas) }\end{array}$ & 2 & 1 & 0 \\
\hline $\begin{array}{l}\text { L. Hacer actividades extenuantes (como caminata en áreas na- } \\
\text { turales, jardinear, mover objetos pesados, andar en bicicleta, } \\
\text { ejercicios de baile, ejercicios de fuerza, etc.) }\end{array}$ & 2 & 1 & 0 \\
\hline
\end{tabular}

Total:

\section{Referencias}

1. Kalache A, Kickbusch I. A global strategy for healthy ageing. World Health 1997; 50 (4): 4-5.

2. Olivares PR, Gusi N, Prieto J, Hernández-Mocholi MA. Fitness and health-related quality of life dimensions in community-dwelling middle aged and older adults. Health Qual Life Outcomes 2011; 9: 117.

3. Rikli RE, Jones CJ. The reliability and validity of a 6-minute walk test as a measure of physical endurance in older adults. J Aging Phys Act 1998; 6: 363-75.

4. Rikli RE, Jones CJ. Development and validation of criterion-referenced clinically relevant fitness standards for maintaining physical independence in later years. The Gerontologist 2012; 53 (2): 255-67.

5. Sardinha LB, Santos DA, Marques EA, Mota J. Criterion-referenced fitness standards for predicting physical independence into later life. Exp Gerontol 2014.

6. Muñiz J, Elosua P, Hambleton RK. Directrices para la traducción y adaptación de los tests: segunda edición. Psicothema 2013; 25 (2): 151-7.

7. Gusi N, Badia X, Herdman M, Olivares PR. [Translation and cultural adaptation of the Spanish version of EQ-5D-Y questionnaire for children and adolescents]. Atención Primaria/Sociedad Española de Medicina de Familia y Comunitaria 2009; 41 (1): 19-23. 
8. Conrad F. Verbal reports are data! A theoretical approach to cognitive interviews. 1999. Proceedings of the Federal Committee on Stadistical Methodology Research Conference. Arlington, Virginia. Consultado el 17 de septiembre de 2003.

9. Herdman M, Fox-Rushby J, Badia X. 'Equivalence'and the translation and adaptation of health-related quality of life questionnaires. Qual Life Res 1997; 6 (3): 237-47.

10. Solís CLB, Arrioja SG, Manzano AO. Índice de Barthel (IB): Un instrumento esencial para la evaluación funcional y la rehabilitación. Plasticidad y restauración neurológica 2005; 4 (1-2): 81-5.

11. Cruz Jentoft A. El índice de Katz. Rev Esp Geriatr Gerontol 1991; 26 (5): 338-48.

12. Hartigan I. A comparative review of the Katz ADL and the Barthel Index in assessing the activities of daily living of older people. Int J Older People Nurs 2007; 2 (3): 204-12.

13. Quiroga P, Albala Brevis CH, Klaasen G. [Validation of a screening test for age associated cognitive impairment, in Chile]. Rev Med Chile 2004; 132 467-78.

14. Little A, Hemsley D, Volans P, Bergmann K. The relationship between alternative assessments of self care ability in the elderly. Br J Clin Psychol 2011; 25 (1): 51-9.

15. Dorevitch M, Cossar R, Bailey F, Bisset T, Lewis S, Wise $\mathrm{L}$, et al. The accuracy of self and informant ratings of physical functional capacity in the elderly. J Clin Epidemiol 1992; 45 (7): 791-8.

16. Stineman MG, Shea JA, Jette A, Tassoni CJ, Ottenbacher KJ, Fiedler R, et al. The Functional Independence Measure: tests of scaling assumptions, structure, and reliability across 20 diverse impairment categories. Arch Phys Med Rehabil 1996; 77 (11): 1101-8.

17. Bowen M, Rowe M, Hart-Hughes S, Barnett S, Ji M. Characteristics of and Barriers to Functional Status Assessment in Assisted Living. Res Gerontol Nurs 2015; $1-11$.

18. Ribeiro DKdMN, Lenardt MH, Michel T, Setoguchi LS, Grden CRB, de Oliveira ES. Fatores contributivos para a independência funcional de idosos longevos. Rev Esc Enferm USP 2015; 49 (1): 89-95.

19. Lazcano B. Evaluación geriátrica multidimensional. En: Rodríguez G, Lazcano B, editors. Práctica de la Geriatría. 2 ed. México: McGraw-Hill Interamericana; 2007. p. 83-104. 